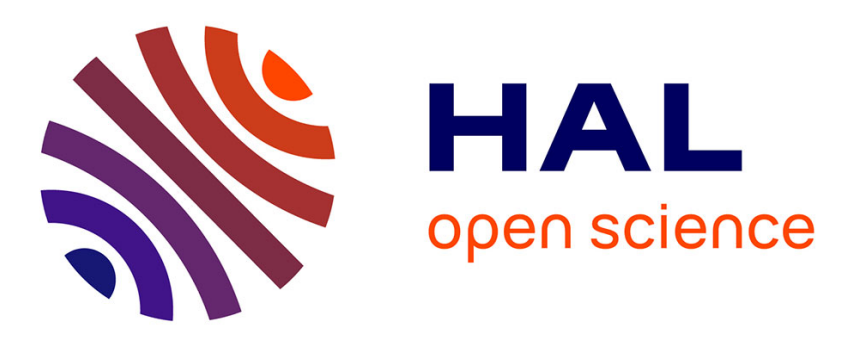

\title{
Automating the measurement of physiological parameters: a case study in the image analysis of cilia motion
}

Élodie Puybareau, Hugues Talbot, E Bequignon, B Louis, G Pelle, J.-F

Papon, A Coste, Laurent Najman

\section{To cite this version:}

Élodie Puybareau, Hugues Talbot, E Bequignon, B Louis, G Pelle, et al.. Automating the measurement of physiological parameters: a case study in the image analysis of cilia motion. IEEE International Conference on Image Processing (ICIP), Sep 2016, Phoenix, United States. 10.1109/ICIP.2016.7532556 . hal-01332942

\section{HAL Id: hal-01332942 \\ https://hal.science/hal-01332942}

Submitted on 16 Jun 2016

HAL is a multi-disciplinary open access archive for the deposit and dissemination of scientific research documents, whether they are published or not. The documents may come from teaching and research institutions in France or abroad, or from public or private research centers.
L'archive ouverte pluridisciplinaire HAL, est destinée au dépôt et à la diffusion de documents scientifiques de niveau recherche, publiés ou non, émanant des établissements d'enseignement et de recherche français ou étrangers, des laboratoires publics ou privés. 


\title{
AUTOMATING THE MEASUREMENT OF PHYSIOLOGICAL PARAMETERS: A CASE STUDY IN THE IMAGE ANALYSIS OF CILIA MOTION
}

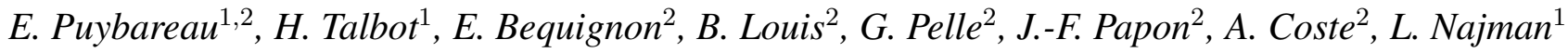 \\ Université Paris-Est, ${ }^{1}$ ESIEE, LIGM, UMR CNRS $8049 ;{ }^{2}$ Inserm U955, CNRS ERL 7240; France.
}

\begin{abstract}
As image processing and analysis techniques improve, an increasing number of procedures in bio-medical analyses can be automated. This brings many benefits, e.g improved speed and accuracy, leading to more reliable diagnoses and follow-up, ultimately improving patients outcome. Many automated procedures in bio-medical imaging are well established and typically consist of detecting and counting various types of cells (e.g. blood cells, abnormal cells in Pap smears, and so on). In this article we propose to automate a different and difficult set of measurements, which is conducted on the cilia of people suffering from a variety of respiratory tract diseases.

Cilia are slender, microscopic, hair-like structures or organelles that extend from the surface of nearly all mammalian cells. Motile cilia, such as those found in the lungs and respiratory tract, present a periodic beating motion that keep the airways clear of mucus and dirt.

In this paper, we propose a fully automated method that computes various measurements regarding the motion of cilia, taken with high-speed video-microscopy. The advantage of our approach is its capacity to automatically compute robust, adaptive and regionalized measurements, i.e. associated with different regions in the image. We validate the robustness of our approach, and illustrate its performance in comparison to the state-of-the-art.
\end{abstract}

Index Terms - frequency analysis, periodic motion, biomedical imaging, mathematical morphology, image stabilization.

\section{INTRODUCTION}

Muco-ciliary clearance is a crucial mechanism of defense against aerial environmental attacks such as micro-organisms or pollution. This clearance is achieved by the coordinated beating of the cilia covering the nasal epithelium. Cilia motility impairment can be either of genetic (primary ciliary dyskinesia) or acquired origin due to environmental attacks and may entailing chronic diseases such as chronic sinusitis and bronchitis. It is of interest for practitioners to evaluate ciliary beating motion (CBM), in particular the cilia measurements and the beating frequency, easily, robustly and reliably. The estimation of ciliary beating frequency has been a research topic since the middle of the 20th century. One of the first method of reference for measurement of cilary beating frequency was proposed in 1962 and used a photo-sensitive cell [1]. Stroboscopic methods have been replaced by more accurate techniques that use photomultiplier, photodiode and high-speed imaging. Those methods are described and compared in [2]. Analysis via high-speed video-microscopy is now considered the most accurate method. Hence, the most commonly used technique today for evaluating ciliary function in human being consists of collecting ciliated cells from nasal or tracheobronchial surface mucosa, to observe them under a microscope and

Thanks to CNRS and University Paris-Est for its CILIOLA starting grant to record their motion via high-speed video acquisition. Evaluation, via these records, of ciliary beating patterns was reported helpful in the diagnosis of primary ciliary dyskinesia $[3,4,5]$.

In clinical research, there exist several methods that estimate ciliary beating motion, but typically only the beating frequency, and nearly all are semi-automated. Cinematic analyzis [6] manually counts the number of frames required to complete 10 ciliary beat cycles. It is a time-consuming and user-dependent method, which has to be repeated several times to obtain a reliable result.

Kymograph analysis [7, 8] is a "linescan" method where grey level variations over a line drawn by the user are analyzed semiautomatically. This method is sensitive to illumination, vibrations, proper cell motion and depends on the location of the line, the shape of the ciliated cells, as such it is only really suitable for straight cell borders. It is also user-dependent.

In the literature, only a few articles tackle the CBM problem. In [9] we presented an automated, region-based solution to measure only the cilia beating frequency using optical flow. A semi automated method also using optical flow is proposed in [10]. In that article, optical flow features are used to distinguish between healthy and unhealthy cells. Unfortunately it is currently not possible to compare the flow features with clinical measurements and so these features have limited diagnostic capability.

In the current paper, we propose a fully automated approach for the analysis of cilia motion that adapts to the cell shape. Our approach is an improvement on the straight linescan techniques coupled with cilia segmentation, which we term "curvescan". In the following sections we present the material and methods; the preprocessing steps; our proposed adaptive curvescan technique; our results and discussion; and finally a conclusion and future work.

\section{MATERIAL AND METHODS}

Cilia are tiny structures of $6 \mu \mathrm{m}$ in length and $0.3 \mu \mathrm{m}$ in diameter. To be able to observe them, ciliated cells were taken from nasal biopsies and placed under a high-power bright field optical microscope. Sequences were acquired with a spatial resolution of $0.13 \mu \mathrm{m}$ and a temporal resolution of 356 frames per second with a high speed camera. The high speed camera exerts a grid-like sensor pattern which degrades the image. Since cells have to be kept in liquid serum to stay alive, the entire cells move due to their beating cilia. Artefactual motion of the whole field of view can sometime be observed due to vibrations.

We note that since the origin of the cilia motion is biological in nature, it is not perfectly periodic. The frequency of the beating may vary slightly over a short timespan. Also different cilia in the same spatial group may beat at different frequencies. For these reason, we refer to the cilia motion as pseudo-periodic. 


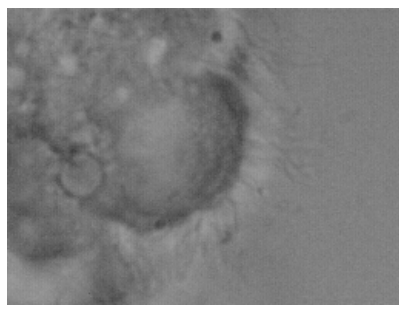

(a)

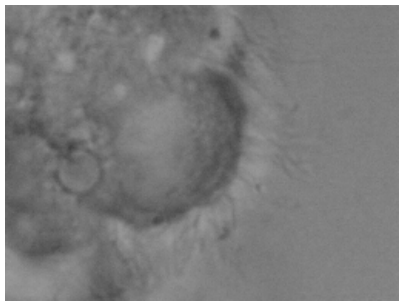

(c)

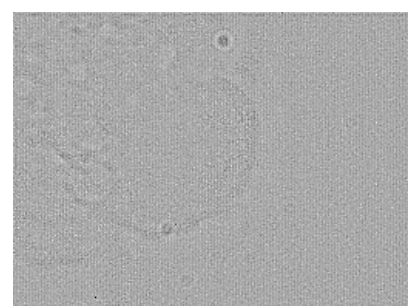

(b)

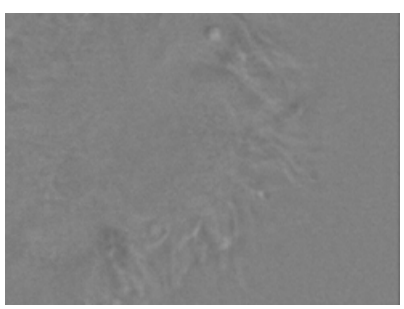

(d)
Fig. 1. Removal of sensor pattern. (a) is the initial frame $I$ of the sequence $\mathcal{S}$, (b) is the sensor pattern obtained by subtracting $\mathcal{G}_{\sigma=1}(\overline{\mathcal{S}})$ to the average $\overline{\mathcal{S}}$, (c) is the result $I_{\text {clear }}$ of the subtraction between the sequence and the sensor pattern $I-\left(\overline{\mathcal{S}}-\mathcal{G}_{\sigma=1}(\overline{\mathcal{S}})\right)(\mathrm{d})$ is the extraction of the moving parts of the sequence $I_{\text {mov }}=I_{\text {reg }}-\overline{\mathcal{S}}_{3}$, using registered images $I_{\text {reg }}$.

\section{PRE-PROCESSING STEPS}

Let $\mathcal{S}$ be the sequence acquired under the microscope. We used a pre-processing similar to [11]. It consists of 3 main steps:

\section{Sensor pattern removal:}

$$
\forall I \in \mathcal{S}: I_{\text {clear }}=I-\left(\overline{\mathcal{S}}-\mathcal{G}_{\sigma=1}(\overline{\mathcal{S}})\right)
$$

where $\mathcal{G}_{\sigma=1}$ is a gaussian filter with standard deviation $\sigma=1$ and $\overline{\mathcal{S}}$ is the average of the sequence $\mathcal{S}$. We called $\mathcal{S}_{2}$ the sequence of the resulting $I_{\text {clear }}$.

Sequence stabilization: To register the sequence, we are looking for a rotation $\mathcal{R}=\left(\begin{array}{c}\cos \theta \sin \theta \\ -\sin \theta \cos \theta\end{array}\right)$ and a translation $\mathcal{T}=\left(d_{x}, d_{y}\right)$ such that;

$$
\forall(x, y) \in I_{\text {clear }}: I_{\text {reg }}(x, y)=I_{\text {clear }}\left(x^{\prime}, y^{\prime}\right)
$$

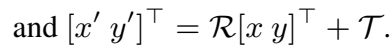

We estimate $\mathcal{R}$ and $\mathcal{T}$ using a robust iterated regression :

$$
P_{1}=P_{2} \times \mathcal{R}+\mathcal{T}
$$

where $P_{1}$ and $P_{2}$ are matching sets of points, extracted using SIFT [12] or SURF [13]. We call $\mathcal{S}_{3}$ the resulting stabilized sequence of $I_{\text {reg }}$

Motion zone estimation: We detect the zones with motion using

$$
\forall I_{\text {reg }} \in \mathcal{S}_{3}: I_{\text {mov }}=I_{\text {reg }}-\overline{\mathcal{S}}_{3}
$$

$\mathcal{S}_{4}$ is the sequence of $I_{m o v}$. These steps are illustrated on Fig. 1.

\section{ADAPTIVE CURVESCAN}

Because cilia beat in 3D, they come in and out of focus during their motion. They also beat in groups. This makes tracking an individual cilium very challenging. The idea of the "linescan" technique is to only observe the variations of intensity in a given narrow, elongated region (typically a line) encompassing the perceived cilia motion. Since the cilia motion is pseudo-periodic, so should be the intensity variation. Here we seek to specify this scanning region automatically, and we do not limit ourselves to a linear region. Cilia located on biopsy cells are rooted along the surface of the cell. Contrary to the experimental conditions of [8], where cultured ciliated cells are located on an even surface, the beating extremity of cilia form a curve. Since cilia may be of varying lengths, it is useful to consider not just a single curve but several from the root to the tip of the beating region. By analogy to the linescan technique, we call this new one the adaptive curvescan.

\subsection{First step: motion zone segmentation}

After pre-processing, beating cilia are the only moving part in the sequence. The temporal variance $\mathcal{V}$ of this sequence (Fig. 2.b) thus highlights the zones with motion.

$$
\mathcal{V}=\mathcal{G}_{\sigma=1} * \sum_{t=1}^{L-1}\left(\nabla_{t} I-\frac{1}{L-1} \sum_{t=1}^{L-1} \nabla_{t} I\right)^{2},
$$

where $\nabla_{t} I=I_{t-1}-I_{t}$ is the temporal gradient. We use a classical watershed-based morphological procedure [14] to segment the different areas of motion. The markers are selected from $\mathcal{M}$, a thresholding of a smoothed dilated image of the variance:

$$
\mathcal{M}=\left(\mathcal{G}_{\sigma=5}\left(\delta_{\mathcal{B}_{3}}(\mathcal{V})\right)\right)_{>0.3 * \max (\mathcal{V})}
$$

where $\delta_{\mathcal{B}_{3}}(I)$ is the dilation of $I$ by an Euclidian ball $\mathcal{B}$ of radius 3 . $I_{>\theta}$ denotes the thresholding of image $I$ above $\theta$.

Internal markers are provided by $\varepsilon_{\mathcal{B}}(\mathcal{M})$ and external marker by $\varepsilon_{\mathcal{B}_{3}}(X \backslash \mathcal{M})$, where $\varepsilon_{\mathcal{B}}$ is the erosion by an Euclidian ball $\mathcal{B}$ and $X$ is the image domain.

The watershed of the variance leads to a mask denoted by $\mathcal{M}_{L}$. Figure 2 shows an example of such a mask, in which all the connected components are distinguished by a different (color) label.

\subsection{Second step : specifying the scan curves}

Each segmented motion zone $a$ of $M_{L}$ is smoothed by a closing followed by an opening of radius 15 , resulting in $a_{f}=\gamma_{\mathcal{B}_{15}} \varphi_{\mathcal{B}_{15}}(a)$, where $\gamma$ is the opening and $\varphi$ the closing. An interior Euclidean distance map [15] $D_{f}$ is then computed from $a_{f}$. The value of 15 was chosen to correspond to a diameter smaller than the smallest cilia, so they are all preserved by the filtering. This filtering simplifies the region contours, removes regions that are too small to contain cilia, separates regions containing different groups of cilia and guarantees that the first 15 level lines of $D_{f}$ are connected. Each level-line of $D_{f}$ allows us to "unroll" the region $a_{f}$ at a different distance from the ciliated cell (see Fig. 3.a). Starting from an arbitrary point of a level line $L_{v}$ at distance $v$ in $D_{f}$, we follow this curve around $a_{f}$ in an 8-connected fashion, recording the pixel coordinates as we go. We stop when we encounter the edge of the image or the initial point after one loop. We create a new image $I_{L_{v}}$ of size (length of the curve $) \times($ number of frames in the sequence $)$, where each column $m$ corresponds to the grey level values of $L_{v}$ in the frame $m$. 


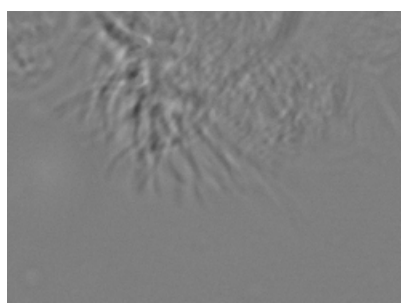

(a)

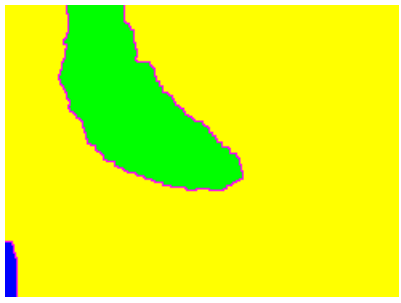

(c)

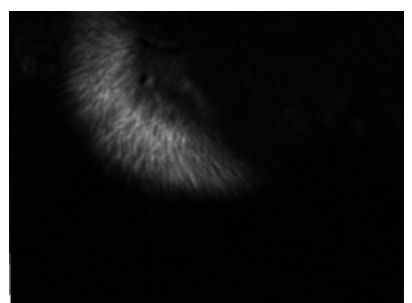

(b)

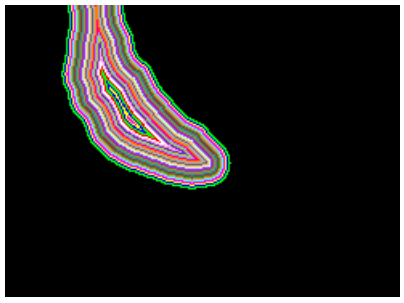

(d)
Fig. 2. Adaptative curvescan. (a) Initial frame from $\mathcal{S}_{4}$. (b) Variance $\mathcal{V}$ of $\mathcal{S}_{4}$. (c) Mask $\mathcal{M}_{L}$ of the zones of movement, obtained with a watershed of (b). (d) Distance map $D_{f}$ inside the green zone of (c), yielding concentric lines.

$$
I_{L_{v}}[n, m]=I_{m o v}^{m}\left[\mathcal{C}_{v}[n]\right]
$$

Where $I_{\text {mov }}^{m}$ corresponds to the frame number $m$ of $I_{m o v}, \mathcal{C}_{v}[n]=$ $\left[n^{\prime}, m^{\prime}\right]$ is the coordinates of the point number $n$ of the line $L_{v}$ in $I_{m o v}^{m}$. We subtracted the output of a spatial median filter with a window size of $11 \times 11$ to eliminate illumination variation and only retain thin objects. We denote $I_{v}$ this resulting image (see Fig. 3.b).

\subsection{Parameters estimation}

The purpose of our method is to allow the estimation of several parameters that are discriminant for diagnosis. In this section, we propose procedures for frequency estimation and cilia length measurement.

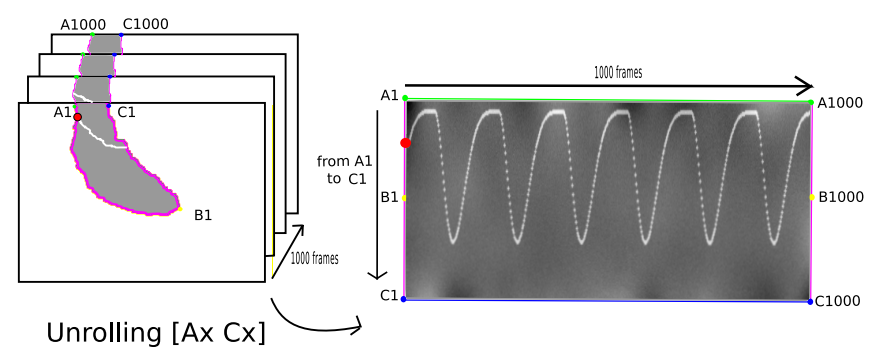

(a)

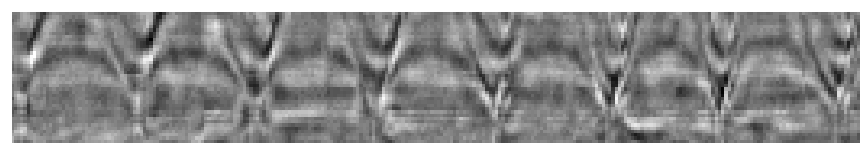

(b)

Fig. 3. Example of grey level extraction.(a) Schematic example of unrolling of the first line in distance map (b) $I_{L v}^{\text {med }}\left(=I_{v}\right.$ ) is an example of curvescan result after median filtering subtraction.

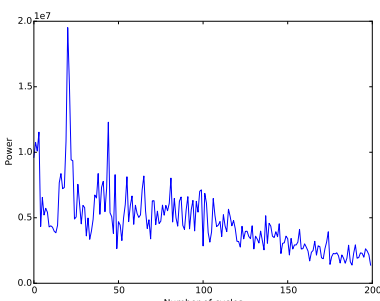

(a)

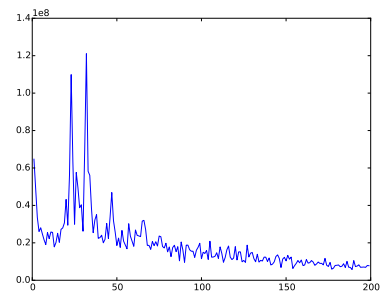

(b)
Fig. 4. Power spectra examples (a) a single frequency present, with harmonics, (b) Two distinct frequencies present.

Frequency estimation We estimated the power spectrum $\mathcal{S}=|\mathcal{F}|^{2}$ by a power spectral density method, exemplified in [8]. It consists of computing the Fourier transform $\mathcal{F}_{n}$ on each line $n$ of $I_{v}$ and averaging the square modulus of all the $\mathcal{F}_{n}$. The cilia beating frequency was then estimated using a parabolic approximation of the main peak and its potential neighbors, in the range of plausible frequencies for cilia (i.e. $0-30 \mathrm{~Hz}$ ).

$$
\begin{gathered}
\forall k \in[0, M-1]: \mathcal{F}_{n}(k)=\sum_{g=0}^{M-1} I_{v}[n, g] e^{-j 2 \pi \frac{g k}{M}} \\
\mathcal{S}(k)=|\mathcal{F}(k)|^{2}=\frac{1}{N} \sum_{n=0}^{N-1}\left|\mathcal{F}_{n}(k)\right|^{2}
\end{gathered}
$$

where $M$ is the number of columns of $I_{v}$ (number of frames of the sequence), and $N$ is the number of pixels of $L_{v}$. For each sequence, we estimated the cilia beating frequencies over the level lines $L_{v} 2,7$ and 14 of $D_{f}$, which are all connected by construction thanks to the filtering of section 4.2, and we took the average as our estimation. Note that the fact that different level lines have differing length has no bearing on the frequency estimation since the time axis has the same length. Also note that we do not need to discriminate on the image $I_{v}$ along the vertical axis for portions that correspond to zones close to the root or near the tip of the cilia, since by continuity of the cilia, the pseudo-periodic motion occurs consistently.

Cilia length measurement The regions of cilia motion segmented in section 4.1 represent the zones where cilia are located. The length $l$ in $\mu \mathrm{m}$ of cilia are represented by the width of the mask. In order to obtain precise measurements, we computed a distance map $D_{f}^{Q}$ on labels of $M_{l}$ using a quadratic Euclidian distance [15]. The maximum value of $D_{f}^{Q}$ inside the region of interest is the square of the radius of the largest inscribed disk, and thus using this value we can obtain a good approximation of the width of the region, and therefore of the length of the cilia beating in it, by using the following formula:

$$
l=\sqrt{\max \left(D_{f}^{Q}\right)} \times 2 \times 0.13
$$

where 0.13 is the spatial resolution ( 1 pixel $=0.13 \mu \mathrm{m})$.

\section{RESULTS AND VALIDATIONS}

Frequency We computed our curvescan results on 11 annotated sequences of beating cilia. We tested our method on cilia exhibiting a variety of beating frequencies, between $6 \mathrm{~Hz}$ and $14 \mathrm{~Hz}$. As stated above, for each sequence, we measured frequencies from 3 different level curves of the distance map, one at the border, one in the center and one in between them. We hence had 33 measurements for 11 sequences. 


\begin{tabular}{|c|c|c|}
\hline & Mean error & Standard deviation on error \\
\hline Single line & 0.0223 & 0.0207 \\
Averaged frequency & 0.0219 & 0.0162 \\
\hline
\end{tabular}

(a)

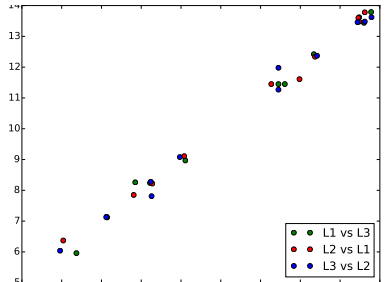

(b)

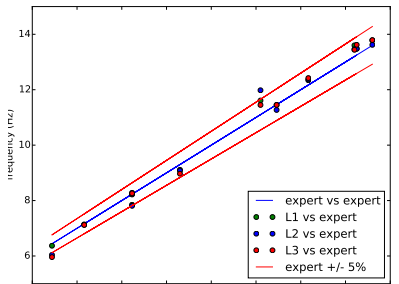

(c)
Fig. 5. Validations of our method for frequency estimation. (a) Mean and standard deviation of our error measurement (b) Validation of the robustness : frequencies estimated on several lines of $D_{f}^{Q}$ are similar. (c) Validation of the accuracy : frequencies obtained with our method correspond to ground truth.

The frequency analysis of the power spectrum may reveal several frequencies present in a region. It may be a single real frequency and its harmonics, or several similar frequencies corresponding to cilia group beating at different frequencies (see Fig. 4). In all cases we only considered the frequency with the highest power in the spectrum.

We validated our results in two steps: we first checked the robustness of the method by comparing the values obtained for each line (see Fig. 5(b)). Then we validated the accuracy of our method by comparing our frequencies with those carefully estimated by experts (see Fig. 5(c)). We measured the average error rate in the classical way with the formula AverageError $=\frac{1}{J} \sum_{j=1}^{J} \frac{\left|m_{j}-t_{j}\right|}{t_{j}}$ with $m_{j}$ our measurement and $t_{j}$ the corresponding ground truth .

We measured the error rate of each measurement separately first, and then the error rate of the averaged estimations. Results obtained are shown in Fig 5(a).The mean errors are similar, about 2.2\%.

Among our 33 measurements, 30 (or 91\%) are within the confidence interval of $95 \%$. However, if we consider the averaged frequency estimations and compare them to the expert estimations, the main frequency is estimated correctly in $100 \%$ of sequences. These results are promising for both the robustness and accuracy of our proposed method.

Cilia Length We computed cilia length measurement on our sequences using the method described in section 4.3. A unique value is obtained for each sequence. An expert manually measured the length of cilia in a region. Their manual measurement was repeated 3 times. We took the average as ground truth for our validations.

We compared our measurements in two different ways (see Fig 6). The first is the equivalent of the frequency validation: we considered the expert measurement as perfect and we measured the AverageError rate. In this way, we obtained an error rate of $2.13 \%$, with one measurement out of 11 outside the $95 \%$ confidence interval and a standard deviation on the error of $2.91 \%$. The second way is the total least square regression method, which seeks a proportionality factor between the manual and automated measurements, taking into account the fact that there could be variability in the expert measurements too. With this method, assuming equal variance between the automated and expert measurements, we achieved an error rate of only $0.8 \%$. The real error rate may be in between these two values. In any case these error rates are reasonably low and so our results are again promising.

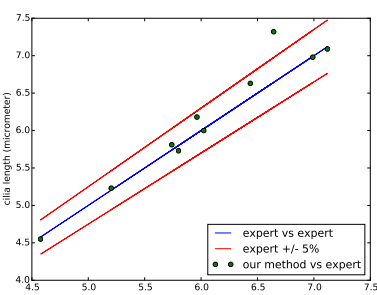

(a)

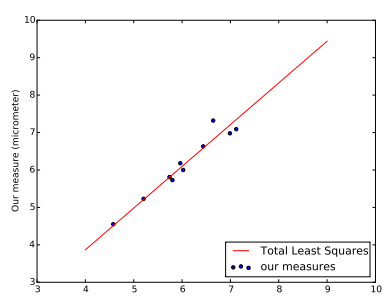

(b)

\begin{tabular}{|c|c|c|}
\hline & Mean error & Standard deviation on error \\
\hline Classical & 0.0213 & 0.0291 \\
TLS & 0.0080 & - \\
\hline
\end{tabular}

(c)

Fig. 6. Validations of our method for cilia length measurement. (a) Validation of the robustness : frequencies estimated on several lines of $D_{f}$ are similar. (b) Validation of the using expert variation with total least squares regression method yields a line which fits our measurements better. (c) Error estimation with the two methods of validation : our mean error may lay somewhere between $0.08 \%$ and $2.13 \%$.

\section{DISCUSSIONS AND CONCLUSION}

We have proposed a novel method for the automated analysis of beating cilia, which are of crucial importance for patients suffering from various forms of severe respiratory diseases. Our method is capable of analyzing entire images consisting of several beating regions. We use the shape of the beating regions to estimate the length of the cilia. Each region of beating cilia may include the presence of multiple beating frequencies (see Fig. 4). We have shown that our estimations are precise, robust and repeatable by validating them against a human observer.

Our method currently records only the main frequency in a beating region but can be easily extended to record all relevant frequencies. We are working on a new segmentation method taking frequency into account to obtain more homogeneous and more precisely defined beating regions. At present we only estimate fairly simple characteristics, however our curvescan results opens the way for more interesting ones. We are currently working on estimating the beating amplitude, regularity, symmetry, and so on. The idea is to analyze the tracks of cilia (see Fig. 7) in the curvescan. With respect to this diagram, we are already measuring $\mathrm{T}_{x}$ and a subset of $\mathrm{D}_{x}$, the distance crossed by cilia. For a more complete set of measurements, we would need to estimate $\mathrm{A}_{x}$ corresponding to the amplitude of beating cilia, and $\mathrm{P}_{x}$ the pause duration in the cilia motion. The combination of these parameters with $\mathrm{T}_{x}$ and the cilia length that we already measure, will provide most of the parameters necessary for disease characterization currently used in clinical practice [5].

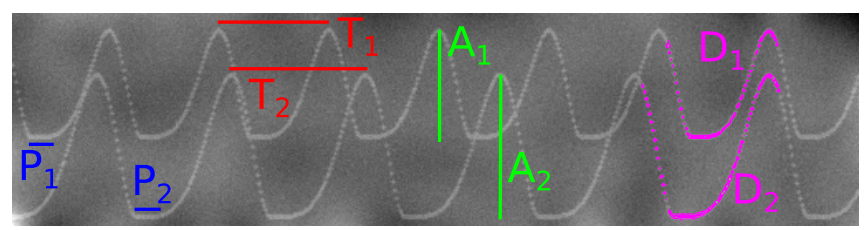

Fig. 7. Ilustration of future work, on synthetic image where we can see two patterns. 


\section{REFERENCES}

[1] T. Dalhamn and R. Rylander, "Frequency of ciliary beat measured with a photo-sensitive cell," Nature, vol. 196, pp. 592$593,1962$.

[2] C. O'Callaghan, K. Sikand, and M. Chilvers, "Analysis of ependymal ciliary beat pattern and beat frequency using high speed imaging: comparison with the photomultiplier and photodiode methods," Cilia, vol. 1, no. 1, 2012.

[3] A. Barbato, T. Frischer, C.E. Kuehni, D. Snijders, I. Azevedo, G. Baktai, L. Bartoloni, E. Eber, A. Escribano, E. Haarman, et al., "Primary ciliary dyskinesia: a consensus statement on diagnostic and treatment approaches in children," European Respiratory Journal, vol. 34, no. 6, pp. 1264-1276, 2009.

[4] W. A. Stannard, M. A. Chilvers, Andrew R Rutman, C. D. Williams, and C. O'Callaghan, "Diagnostic testing of patients suspected of primary ciliary dyskinesia," American journal of respiratory and critical care medicine, vol. 181, no. 4, pp. 307-314, 2010.

[5] J.-F. Papon, L. Bassinet, G. Cariou-Patron, F. Zerah-Lancner, A.-M. Vojtek, S. Blanchon, B. Crestani, S. Amselem, A. Coste, B. Housset, et al., "Quantitative analysis of ciliary beating in primary ciliary dyskinesia: a pilot study," Orphanet journal of rare diseases, vol. 7, no. 1, pp. 78, 2012.

[6] M. A. Chilvers and C. O'Callaghan, "Analysis of ciliary beat pattern and beat frequency using digital high speed imaging: comparison with the photomultiplier and photodiode methods," Thorax, vol. 55, no. 4, pp. 314-317, 2000.

[7] R.T. Doyle, T. Moninger, N. Debavalya, and W.H. Hsu, "Use of confocal linescan to document ciliary beat frequency," Journal of Microscopy, vol. 223, pp. 159-164, 2006.

[8] O. Meste, F. Brau, and A. Guyon, "A New Method for the Robust Estimation of the Motile Cilia Beating Frequency," Tech. Rep. hal-00806681, University of Nice-Sophia Antipolis, 2013.

[9] E. Puybareau, H. Talbot, G. Pelle, B. Louis, J.-F. Papon, A. Coste, and L. Najman, "A regionalized automated measurement of ciliary beating frequency," in Biomedical Imaging (ISBI), IEEE 12th International Symposium on. IEEE, 2015, pp. 528-531.

[10] S. P. Quinn, M. J. Zahid, J. R. Durkin, R. J. Francis, C. W. Lo, and S. C. Chennubhotla, "Automated identification of abnormal respiratory ciliary motion in nasal biopsies," Science Translational Medicine, vol. 7, no. 299, 2015.

[11] E. Puybareau, H. Talbot, and M. Leonard, "Automated heart rate estimation in fish embryo," in Image Processing Theory, Tools and Applications (IPTA), International Conference on, Orleans, France, Nov. 2015, pp. 379-384.

[12] D. G. Lowe, "Object recognition from local scale-invariant features," in IEEE International Conference on Computer Vision, Kerkyra, Greece, September 1999, vol. 2, pp. 1150-1157.

[13] H. Bay, T. Tuytelaars, and L. Van Gool, "Surf: Speeded up robust features," in Computer vision-ECCV 2006, pp. 404 417. Springer, 2006.

[14] F. Meyer and S. Beucher, "Morphological segmentation," Journal of Visual Communication and Image Representation, vol. 1, no. 1, pp. 21-46, Sept. 1990.
[15] T. Saito and J.I. Toriwaki, "New algorithms for euclidean distance transformation of an $\mathrm{N}$-dimensional digitized picture with applications," Pattern recognition, vol. 27, no. 11, pp. 1551-1565, 1994. 